\title{
Association Between Latency of Dermatomal Sensory-Evoked Potentials and Quantitative Radiologic Findings of Narrowing in Lumbar Spinal Stenosis
}

\author{
Dong Chan Yang, $\mathrm{MD}^{1}$, Ho Jun Lee, MD, PhD², Jin-Woo Park, MD, PhD ${ }^{2}$, Kiyeun Nam, MD, $\mathrm{PhD}^{2}$, \\ Shengshu Kim, $\mathrm{MD}^{2}$, Keun-Tae Cho, MD, $\mathrm{PhD}^{3}$, Bum Sun Kwon, MD, $\mathrm{PhD}^{2}$ \\ ${ }^{1}$ Department of Health Service, Gunsan-si Health Center, Gunsan; \\ ${ }^{2}$ Department of Physical Medicine and Rehabilitation, Dongguk University Ilsan Hospital, \\ Dongguk University College of Medicine, Goyang; \\ ${ }^{3}$ Department of Neurosurgery, Dongguk University Ilsan Hospital, Dongguk University College of Medicine, Goyang, Korea
}

Objective To identify the difference of quantitative radiologic stenosis between a normal latency group and an abnormal latency group, and to investigate the association of dermatomal somatosensory-evoked potential (DSEP) with magnetic resonance imaging (MRI) findings of narrowing in patients with lumbar spinal stenosis (LSS).

Methods We retrospectively reviewed the clinical records and P40 latencies of L5 DSEP of 40 patients with unilateral symptoms of LSS at the L4-5 disc level. Quantitative assessments of stenosis in lumbar spine MRI were performed with measurements of the anteroposterior diameter (APD), cross-sectional area (CSA) of the dural sac, ligamentous interfacet distance (LID), CSA of the neural foramen (CSA-NF), and subarticular zone width. Analyses were conducted through comparisons of radiologic severity between the normal and abnormal latency groups and correlation between radiologic severity of stenosis and latency of DSEP in absolute (APD $<10 \mathrm{~mm}$ ) and relative (APD $<13 \mathrm{~mm}$ ) stenosis.

Results The radiologic severities of lumbar stenosis were not significantly different between the normal and abnormal latency groups. In absolute and relative stenosis, latency showed a significant negative correlation with APD ( $\mathrm{r}=-0.539, \mathrm{r}=-0.426)$ and LID $(\mathrm{r}=-0.459, \mathrm{r}=-0.494)$. In patients with relative stenosis, a weak significant positive correlation was found between latency and CSA-NF $(r=0.371, p=0.048)$. LID was the only significant factor for latency $(\beta=-0.930, p=0.011)$.

Conclusion The normal and abnormal DSEP groups showed no significant differences inradiologic severity. The latency of DSEP had a negative correlation with the severity of central stenosis, and LID was an influencing factor.

Keywords Evoked potentials, Magnetic resonance imaging, Spinal stenosis, Electrodiagnosis

Received September 27, 2019; Revised November 2, 2019; Accepted November 19, 2019; Published online September 28, 2020 Corresponding author: Ho Jun Lee

Department of Physical Medicine and Rehabilitation, Dongguk University Ilsan Hospital, 27 Dongguk-ro, Ilsandong-gu, Goyang 10326, Korea. Tel: +8231-961-7460, Fax: +82-31-961-7488, E-mail: hjrhee1@dumc.or.kr

ORCID: Dong Chan Yang (https://orcid.org/0000-0001-7733-4066); Ho Jun Lee (https://orcid.org/0000-0002-1997-2593); Jin-Woo Park (https://orcid. org/0000-0003-4989-2575); Kiyeun Nam (https://orcid.org/0000-0001-6932-6541); Shengshu Kim (https://orcid.org/0000-0003-0410-2410); Keun-Tae Cho (https://orcid.org/0000-0002-8372-7250); Bum Sun Kwon (https://orcid.org/0000-0001-7755-435X).

(a) This is an open-access article distributed under the terms of the Creative Commons Attribution Non-Commercial License (http://creativecommons.org/ licenses/by-nc/4.0) which permits unrestricted noncommercial use, distribution, and reproduction in any medium, provided the original work is properly cited. Copyright ( 2020 by Korean Academy of Rehabilitation Medicine 


\section{INTRODUCTION}

Lumbar spinal stenosis (LSS) is one of the most common degenerative diseases of the spine, especially in old age. LSS has been defined as "buttock or lower-extremity pain, which may occur with or without low back pain, associated with diminished space available for the neural and vascular elements in the lumbar spine" [1]. Disc degeneration, facet degeneration and hypertrophy, degenerative spondylolisthesis, and ligamentum flavum hypertrophy and calcification can cause LSS, alone or in combination [2]. Compression caused by both central stenosis and lateral stenosis is believed to contribute to neurogenic claudication associated with LSS [3]. As stenosis is found in many levels and L4-5 is the most frequently involved level $[4,5]$, the L5 root is most likely to be involved. Despite its high prevalence, there is no generally accepted gold standard method for the diagnosis of LSS. A proper diagnostic method is essential to prevent false-positive results, which can lead to unnecessary treatments [6].

When a clinical impression of LSS is made, the diagnosis is confirmed on the basis of the finding of "narrowing" or "stenosis" of the spine on imaging modalities such as computed tomography or magnetic resonance imaging (MRI). The North American Spine Society states in their guidelines that imaging is the key non-invasive test for LSS, and MRI is the most commonly used imaging method [1]. Nevertheless, its role remains controversial. Wassenaar et al. [7] summarized the diagnostic accuracy of MRI for lumbar disc herniation and spinal stenosis, and suggested that the evidence for the diagnostic accuracy of MRI is inconclusive. Another review article on the radiologic signs of LSS concluded that there is a need for consensus on well-defined, unambiguous radiologic criteria to define LSS [8]. A Delphi survey of radiologic criteria for the diagnosis of LSS concluded that "there are no broadly accepted quantitative criteria and only partially accepted qualitative criteria for the diagnosis of LSS" [9]. Nevertheless, constant efforts have been made for evaluating LSS based on quantitative radiologic findings. In a report dealing with radiologic criteria and parameters for LSS, the anteroposterior diameter (APD) of the spinal canal, cross-sectional area of the dural sac (CSA-DS), ligamentous interfacet distance (LID), and others were included as parameters for central stenosis, and lateral recess height, depth of the lateral recess, and lateral recess angle were included as parameters for lateral stenosis [8,9]. Absolute stenosis is defined as APD of the spinal canal $<10 \mathrm{~mm}$, and relative stenosis is defined as APD $<13 \mathrm{~mm}[3,8]$. Stenosis of the CSA of the spinal canal is defined as CSA-DS of $<100$ or $<130 \mathrm{~mm}^{2}$, and lateral stenosis is defined according to thecutoff values of lateral recess height, as follows: $5 \mathrm{~mm}$, normal (no stenosis); $<3$ $\mathrm{mm}$, highly indicative; and $<2 \mathrm{~mm}$, diagnostic [8]. Many new suggestions have been made to define "stenosis" of the spinal canal and to identify the proper criteria.

To evaluate neurophysiologic deficits, electrodiagnostic methods such as dermatomal somatosensory-evoked potential (DSEP) studies can be used. DSEP offers the possibility of detecting neurophysiologic deficits in LSS, resulting in chronic compression of relatively long segments of dorsal rootlets $[10,11]$. Therefore, they can be used to provide additional information to the neurologic examination when LSS is suspected [12]. However, conventional electrodiagnostic studies have shown varying sensitivity and specificity using clinical reference standards and anatomic reference standards. Therefore, they showed no superior accuracy compared with MRI. This suggests that the evidence of DSEP for the diagnosis of LSS is still insufficient $[4,13]$.

Nevertheless, DSEP was suggested to have a diagnostic value in LSS by representing chronic compression of a root in the spinal canal [10]. Eltantawi et al. [14] reported that DSEP had the highest electrophysiologic abnormality in patients with LSS and a good correlation with symptoms of neurogenic claudication; thus, DSEP had added to the clinical and radiologic assessment of patients with LSS by providing evidence for root dysfunction. Shen et al. [15] reported that the sensitivity and diagnostic concurrence with surgery of nerve root injury following LSS evaluated using DSEP was $95.7 \%$, and the P40 latencies at L4, L5, and S1 in the case group were significantly longer than those in the control group. MRI is suggested as the most appropriate non-invasive test to confirm the presence of anatomic narrowing of the spinal canal or the presence of nerve root impingement [1]. Therefore, we assumed that there will be a significant correlation between neurophysiologic deficits in DSEP studies and anatomic narrowing in MRI in patients with LSS. We hypothesized that prolongation of the DSEP wave may be associated with narrowing of the spinal canal or neural 
foramen on MRI. The objectives of this study were to identify the difference of quantitative radiologic stenosis between a normal latency group and an abnormal latency group, and to investigate the association of L5 DSEP with quantitative radiologic stenosis on MRI in patients with LSS at the L4-5 disc level.

\section{MATERIALS AND METHODS}

\section{Subjects}

We retrospectively reviewed the medical records of patients with LSS at the L4-5 disc level. The inclusion criteria were as follows: unilateral buttock and leg symptoms with claudication confirmed as LSS on MRI, and electrodiagnostic tests including DSEP from January 2010 to December 2016 at the authors' hospital. Ninety patients were identified. Fifty-one patients were excluded to eliminate the possibility of other causes having an influence on the conduction of the waves. The exclusion criteria were as follows: (1) history of lumbar surgery before MRI and DSEP studies ( $n=11)$; (2) presence of polyneuropathy such as diabetic polyneuropathy $(n=26)$; (3) diagnosis of cervical or thoracic myelopathy $(n=3)$; (4) history of spinal cord injury or stroke $(n=2)$; (5) findings of more severe spinal stenosis in L3-4 and L5-S1 $(n=6)$; and (6) APD >13 mm on MRI ( $\mathrm{n}=3$ ). We reviewed the demographic and clinical characteristics including age, sex, height, body mass index, and duration of LSS symptoms in 40 patients after exclusion. The study was approved by the Institutional Review Board of the Dongguk University Ilsan Hospital (No. 2016-52). The informed consent was waived.

\section{DSEP study}

We collected the P40 latencies of L5 DSEPs in both lower extremities. DSEP study was usually performed in L5 dermatomes of both legs to investigate the conduction abnormality (including demyelination and axonotmesis) in the spinal root caused by spinal stenosis at the L4-5 disc level. The DSEP study was performed with a certified machine (Medelec Synergy; Oxford Instruments, Surrey, UK) using a stimulation intensity double or triple to that of the sensory threshold with a pulse duration of $200 \mu \mathrm{s}$ at a rate of $<5 \mathrm{~Hz}$. Stimulation was performed with the cathode located at either the level of the first metatarsophalangeal joint along its medial aspect or in the web space between the first and second digits of the foot. An anode was located about 2 or $3 \mathrm{~cm}$ distal to the placement of the cathode. The ground electrode was located on the ipsilateral patella. Recordings were performed at $\mathrm{Cz}^{\prime}$ and in reference to Fz on the scalp. Two sets of 128 responses were averaged to confirm the consistency of the waves in both lower extremities. P40 latency was detected from the waves for each patient. Instrument parameters were set with an analysis time of $100 \mathrm{~ms}$ and an amplifier sensitivity of 1 or $2 \mu \mathrm{V} /$ div. We used two kinds of criterion of normal P40 latency. For the significant reference, P40 latency exceeding $51.0 \mathrm{~ms}$ was considered as delayed P40 latency [16] and a $>3$ ms difference between the latency on the affected side and the non-symptomatic side was considered to be an abnormal latency difference [17]. Because it has been reported that lumbar DSEP latency has a significant relationship to height, we used the following formula to standardize the data to the average height in our group for statistical comparison [18]:

Standardized lumbar DSEP latencies $=\frac{\text { Lumbar DSEP latencies } \times \text { Average height }}{\text { Individual height }}$.

\section{Measurement of radiologic severity on MRI}

The MRI examination was performed at $1.5 \mathrm{~T}$ using Siemens Avanto equipment (Siemens, Forchheim, Germany), and we selected the axial images (T2-weighted images of MRI) at the level of the L4-5 intervertebral disc for measuring central and lateral recess stenosis. The sagittal images (T2-weighted images) for the neural foramen on the affected side between the L5 and S1 level, where the L5 nerve root exits, were selected for assessing the CSA of the neural foramen (CSA-NF). Thereafter, we captured the images using the Picture Archiving and Communication System. The APD of the spinal canal, LID, and subarticular zone width (SZW) were measured by drawing a straight line between structures and were expressed in millimeters. The other parameters were measured by drawing their outlines with the regions of interest of the PiView (Infinitt, Seoul, Korea) program and were calculated as values in square millimeters.

On MRI, we measured the APD of the osseous spinal canal, CSA-DS, and LID to assess central stenosis; SZW to assess lateral stenosis; and CSA-NF to assess foramen stenosis $[3,6,8]$. LID was defined as the distance between the inner surfaces of the ligamentum flavum on a line connecting the joint space of facet joints at the level of 
the L4-5 intervertebral disc $[7,8,19]$. Its cutoff value for stenosis was $12 \mathrm{~mm}$ in one report and 15 or $16 \mathrm{~mm}$ in other reports [8]. With respect to lateral stenosis, SZW is the distance between the most anterior point of the superior articular facet and the posterior border of the vertebral body [20]. CSA-NF was measured on sagittal images below the pedicle. Because the nerve root is located more cranially than the lower end plate, no space below the line parallel to the lower end plate was included in area measurements [7,8] (Fig. 1).

\section{Statistical analyses}

We compared the differences in clinical characteristics and in radiologic narrowing between the normal and abnormal DSEP latency groups. For these comparisons, we performed the analysis using two reference values. We analyzed the correlation between standardized DSEP latencies and quantitative radiologic findings using Pearson correlation coefficient analysis in patients with absolute stenosis and those with relative stenosis, respectively. After the correlation analysis, backward stepwise regression for each factor, including clinical characteristics and radiologic parameters, was performed to establish the most influential factor for P40 latency. All statistical analyses were performed using SPSS-K version 20.0 (IBM SPSS, Armonk, NY, USA). The level of statistical significance was set at a $\mathrm{p}$-value of $<0.05$.

\section{RESULTS}

DSEP waves with P40 latency were evoked in 29 patients, and no wave was evoked in 10 patients. The average standardized P40 latency was $42.39 \pm 1.44 \mathrm{~ms}$. Abnormal latency was found in $46 \%(n=18)$ of the patients. Comparison between the normal and abnormal latency groups with the reference value (P40 latency $<51 \mathrm{~ms}$ ) showed no significant differences in clinical characteristics and radiologic measurements. Abnormal latency difference was found in $44 \%(n=17)$ of the patients. Another comparison between the normal and abnormal latency difference groups also showed no significant differences (Table 1). In patients with relative stenosis of APD $<13$ $\mathrm{mm}$, standardized P40 latency had a significant negative correlation with the APD (correlation coefficient $[\mathrm{r}]=-0.426$, $\mathrm{p}=0.021)$ and LID ( $\mathrm{r}=-0.494, \mathrm{p}=0.006)$ of the spinal canal, which represent the morphologic severity of central steno-
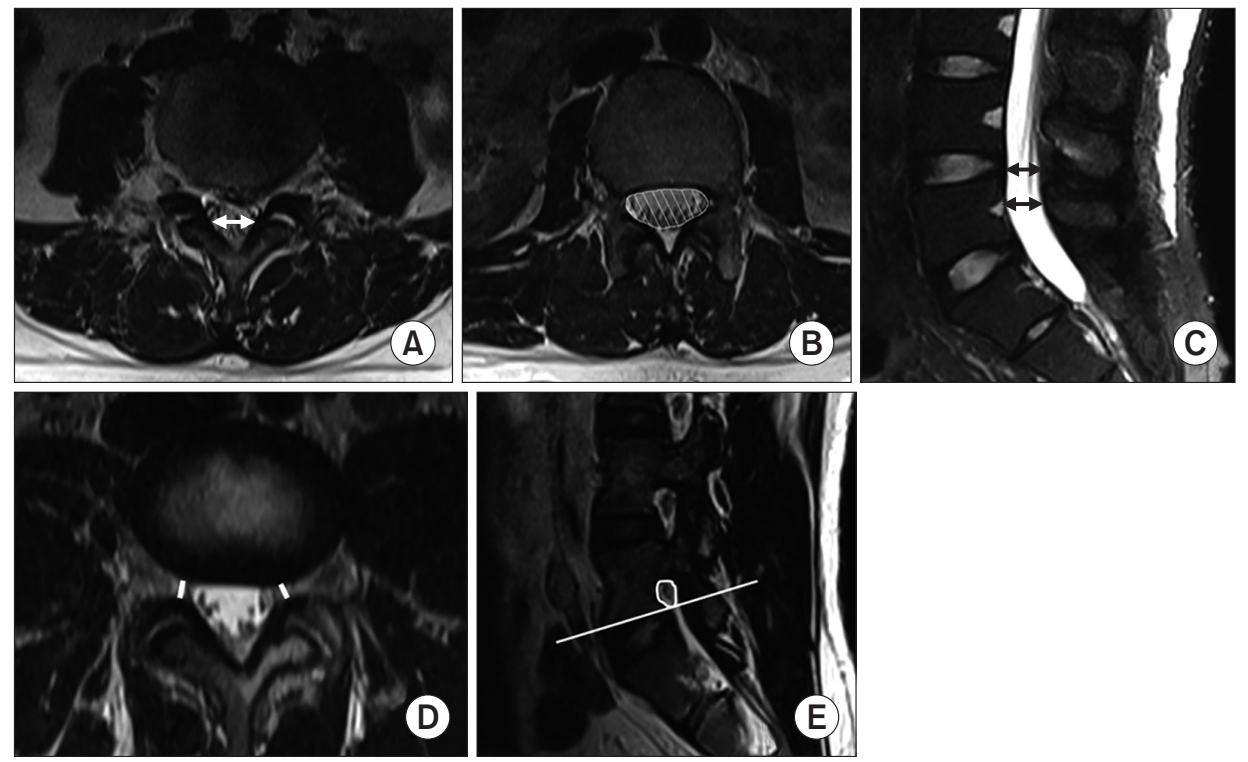

Fig. 1. (A) The white arrow indicates the ligamentous interfacet distance measured between the inner surfaces of ligamentum flavum by connecting the joint space of facet joints. (B) Cross-sectional area of the dural sac is indicated by the white hatched area. (C) The black arrow indicates the anteroposterior diameter of the osseous spinal canal. (D) The minimal width of the subarticular zone was measured at the level of nerve roots using the disc, ligamentum flavum or facet joint as structures that constitute the borders of the neural canal. (E) The cross-sectional area of the neural foramen was measured on sagittal images below the pedicle. Because the nerve root is located more cranially than the lower end plate, no space below the line parallel to the lower end plate was included in area measurements. 


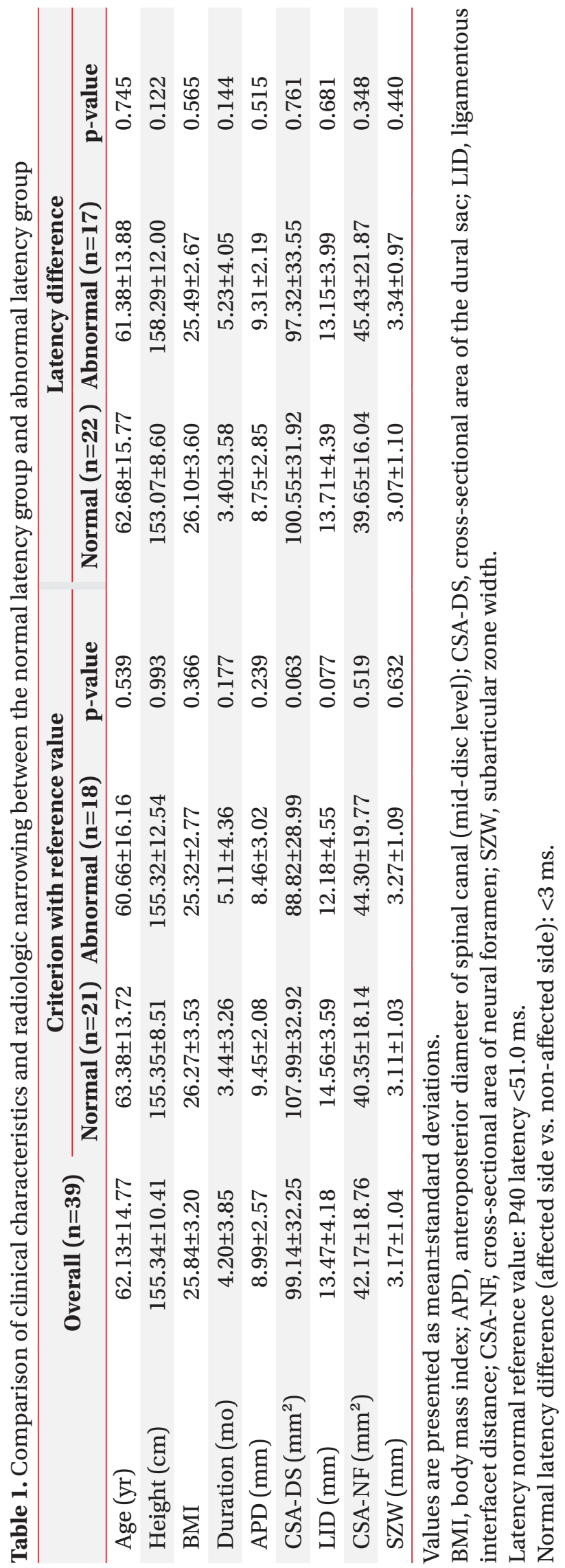

sis. CSA-NF had a significant but weak positive correlation with standardized P40 latency ( $\mathrm{r}=0.318, \mathrm{p}=0.048)$. In patients with absolute stenosis of APD $<10 \mathrm{~mm}$, standardized P40 latency had a more significant negative correlation with APD ( $\mathrm{r}=-0.539, \mathrm{p}=0.007)$ and a less significant negative correlation with LID $(\mathrm{r}=-0.459, \mathrm{p}=0.024)$. However, P40 latency had no significant correlation with CSA-NF $(\mathrm{r}=0.363, \mathrm{p}=0.081)$ (Table 2). In backward stepwise regression analysis between $\mathrm{P} 40$ latency and each factor in all patients with evoked waves, LID was shown to be the only significant factor (regression coefficient $[\beta]=-0.930$, $\mathrm{p}=0.011)$.

\section{DISCUSSION}

To the best of our knowledge, there is no article on quantitative analysis and direct correlation between DSEP and stenosis parameters of MRI in patients with LSS. The diagnostic criteria for LSS are uncertain and consensus among experts is not confirmed. Moreover, imaging studies such as MRI can be expected to indicate the morphologic severity of stenosis. However, the diagnostic validity is unclear. Therefore, the diagnostic value of DSEPs is worth investigating because a delay in DSEP latency can be assumed to physiologically reflect stenosis around the spinal nerve root in the central spinal canal and neural foramen. Therefore, we expected a significant difference in MRI parameters for spinal stenosis between the groups categorized by the severity of DSEP findings, such as the prolongation of latency itself and the difference in latency on the symptomatic and asymptomatic sides. Additionally, we expected that the prolongation or abnormality in DSEP wave latency will have a significant negative correlation with the morphologic severity of stenosis on MRI, which means that the more severe is the stenosis, the more delayed would be the P40 latency. However, the comparison of radiologic parameters between the normal latency group and the abnormal latency group did not show any significant results. Therefore, when diagnosing LSS based on imaging modalities, the additional role of DSEPs seems less valuable. In fact, the diagnostic value of DSEPs is still controversial. The results of this study showed abnormal latency in $46 \%(n=18)$ of all included patients and an abnormal difference in latency in $44 \%$ ( $n=17)$ of all included patients.

DSEP study was suggested to be valuable as an add- 
Table 2. Pearson correlation between $\mathrm{P} 40$ patency and MRI measurements in the evoked wave group ( $\mathrm{n}=29)$

\begin{tabular}{lcccc}
\hline & \multicolumn{2}{c}{ APD $<\mathbf{1 3} \mathbf{~ m m}(\mathbf{n}=\mathbf{2 9})$} & APD $<\mathbf{1 0 ~} \mathbf{~ m m}(\mathbf{n}=\mathbf{2 4})$ \\
\cline { 2 - 5 } & Pearson correlation coefficient & p-value & Pearson correlation coefficient & p-value \\
\hline APD $(\mathrm{mm})$ & -0.426 & $0.021^{*}$ & -0.539 & $0.007^{*}$ \\
CSA-DS $\left(\mathrm{mm}^{2}\right)$ & -0.331 & 0.079 & -0.287 & 0.175 \\
LID $(\mathrm{mm})$ & -0.494 & $0.006^{* *}$ & -0.459 & $0.024^{*}$ \\
CSA-NF $\left(\mathrm{mm}^{2}\right)$ & 0.371 & $0.048^{*}$ & 0.363 & 0.081 \\
SZW $(\mathrm{mm})$ & 0.318 & 0.092 & 0.269 & 0.204 \\
\hline
\end{tabular}

APD, anteroposterior diameter of spinal canal (mid-disc level); CSA-DS, cross-sectional area of the dural sac; LID, ligamentous interfacet distance; CSA-NF, cross-sectional area of neural foramen; SZW, subarticular zone width.

${ }^{*} \mathrm{p}<0.05,{ }^{* *} \mathrm{p}<0.01$.

on diagnostic procedure to imaging studies [14], as well as to be a useful supplementary test for the diagnosis of LSS [21]. Eltantawi et al. [14] reported the high sensitivity of abnormal waves of DSEPs through a comparison with a control healthy group and set clinical symptoms of claudication as a comparative standard. However, they compared only two variables (normal and abnormal latencies without real latency) and did not analyze quantitative data. In patients with LSS, another study reported that tibial somatosensory-evoked potentials (SEPs) were delayed in $78 \%$, pathologic compound muscle action potential reductions were found in $39 \%$, and a pathologically prolonged H-reflex was found in $52 \%$. That study included patients with symptoms in both limbs [12]. A recent review reported that the diagnostic accuracy of DSEPs remains unclear despite the high sensitivity because of the small number of studies and the use of different reference standards. The diagnostic accuracy of DSEPs was reported to have a high or modest sensitivity (78\%-94\%), with no reports about the specificity. Overall, the diagnostic accuracy of other electrodiagnostic tests (electromyography [EMG], nerve conduction study, magnetic stimulation-induced motor conduction time, and selective lumbar root sheath infiltration) was only modest. Paraspinal mapping had a high specificity in two studies and may increase the likelihood of diagnosing LSS. The authors concluded that these electrodiagnostic studies showed no superior accuracy for conventional electrodiagnostic testing compared with MRI [6]. Another recent study reported that needle EMG showed a significant correlation with clinical symptoms, whereas MRI findings did not show a significant correlation in patients with lateral stenosis on MRI. The authors suggested that EMG findings can physiologically reflect stenosis around the nerves, but MRI parameters are not sufficient to explain the multifactorial causes of LSS [22].

However, when we analyzed the correlation between standardized DSEP latencies and quantitative radiologic findings in patients with evoked waves, the APD of the spinal canal and the LID showed significant negative correlations with the P40 latency in both the relative central stenosis and absolute central stenosis groups. In backward stepwise regression for each factor, we observed that LID was the only significant factor affecting P40 latency. We suggest that P40 latency seems to be affected by central stenosis, especially because of ligamentum flavum thickening, which is related to central stenosis. Further, it can be suggested that ligamentum flavum thickening has a greater influence on the dorsal root transporting sensory signals than on the ventral root; thus, it can affect the latency of P40 SEP waves. Another study with 54 patients undergoing decompressive spine surgery after the diagnosis of LSS reported that there was no significant correlation between tibial SEPs and the CSA-DS and the osseous spinal canal on MRI [12]. Liu et al. [18] reported that lumbar SEP study, which was performed with recording at the T12 spinous process and was suggested to be well restricted to the lumbar region, is able to effectively detect neurologic deficits in the lumbar area in patients with LSS. They supposed that cortical SEPs, which are the most commonly used, cannot reflect the true neurologic condition of the lumbar spine and are not very useful in clinical assessment. The findings of these reports are opposite to our results; however, these studies did not include LID as a parameter of central stenosis. Conversely, the results on the relationship between P40 latency and lateral stenosis parameters were beyond our expectation. CSA-NF showed a weak significant posi- 
tive correlation with $\mathrm{P} 40$ latency $(\mathrm{p}=0.048)$ only in relative spinal stenosis, but showed no significant correlation in absolute, more severe, spinal stenosis. We supposed that these findings possibly resulted from technical errors, because measuring neural foramen stenosis was more difficult than measuring central stenosis and lateral stenosis. CSA-NF was measured on sagittal images below the pedicle [20]. Hence, it was rather difficult to select the exact sagittal image cut representing the foraminal zone. Moreover, as the nerve root is located more cranially than the lower end plate, no space below the line parallel to the lower end plate was included [19]. In fact, CSA-NF is not a generally accepted radiologic parameter for lateral stenosis [23]. Semi-quantitative or qualitative radiologic criteria such as the grading system according to the amount of perineural intraforaminal fat, hypertrophic facet joint degeneration, compression of the foraminal zone, foraminal nerve root impingement, and size and shape of the foramen havebeen reported. The grading system of perineural intraforaminal fat showed good reliability, but the other criteria showed variability in intrarater and inter-rater reliability [24]. With respect to SZW, it was measured by drawing a line between the most anterior point of the superior articular facet and the posterior border of the vertebral body [19]. This measurement requires a rather delicate process because even a slight error in measurement may severely affect the latency, as its mean \pm standard deviation was $3.17 \pm 1.00 \mathrm{~mm}$ (Table 1). If a larger number of patients are involved, the impact of a single technical error would be reduced.

This study had some limitations. First, there is no gold standard method for the diagnosis of spinal stenosis. Surgical findings and the clinical results after surgery could be the best diagnostic criteria; however, there were 10 patients who had undergone spinal surgery after the diagnosis and we could not perform the analysis according to surgical findings. Second, this was a retrospective study with insufficient clinical information. As the diagnosis of spinal stenosis was usually based on the clinical impression, the analysis of the relationship of DSEP to clinical characteristics such as pain intensity, claudication severity, and functional scales, such as Oswestry Disability Index, can be valuable. However, because it was a retrospective review, we could not obtain such clinical information and additional analyses including clinical factors, latency of DSEPs, and morphologic severity are needed to identify the diagnostic usefulness of DSEPs in patients with LSS. Third, the number of patients for the analysis was small. We did not routinely perform DSEP studies based on the impression of spinal stenosis or herniated disc disease because of the low sensitivity of needle EMG and nerve conduction study. We only performed a DSEP study in cases in which there was a strong impression of spinal stenosis or a referral from spine surgeons to identify the involvement of the root in LSS. Therefore, the number of patients with DSEP data was small. Fourth, there was no segmental study. Liu et al. [18] reported that lumbar SEPs are able to effectively detect neurologic deficits in the lumbar area in patients with LSS, and cortical SEPs cannot reflect the true neurologic condition of the lumbar spine. However, another study comparing segmental mixed nerve SEPs with DSEPs reported that S1 and L5 DSEP latency showed higher sensitivity and specificity and relatively lower sensitivity and specificity in N25 and N45 latency, and poor sensitivity and specificity in N20 and N10 latency of tibial nerve mixed SEPs [21]. We only performed cortical SEP study with P40 latency, which represents the function of the long sensory tract from the foot to the cortex, because we experienced difficulty in obtaining waves in the segmental study with simultaneous recording at the popliteal fossa, lumbar spine, thoracic spine, and scalp. We attempted to exclude diseases that may have an influence on the long sensory tract, such as myelopathy and distal sensorimotor polyneuropathy.

In conclusion, despite the lack of differences in the radiologic severity of LSS between the normal and abnormal DSEP groups, the latency of DSEPs had a negative correlation with the severity of central stenosis and LID was an influencing factor in the delay of DSEP latency. Further studies about the association between clinical factors and DSEP and the diagnostic value of DSEP will be required.

\section{CONFLICT OF INTEREST}

No potential conflict of interest relevant to this article was reported.

\section{ACKNOWLEDGMENTS}

This work was supported by the Dongguk University 
Research Fund of 2016. Special thanks are due to the biostatistician (Chi-Yeon Lim, PhD) of the Department of Data Management and Statistics Institute, Dongguk University.

\section{AUTHOR CONTRIBUTION}

Conceptualization: Lee HJ. Methodology: Lee HJ, Yang DC, Nam K, Cho KT. Formal analysis: Lee HJ, Yang DC, Park JW. Investigation: Nam K, Yang DC, Kim S. Resources: Lee HJ, Nam K, Park JW. Writing - original draft: Yang DC. Writing - review and editing: Lee HJ. Supervision: Kwon BS. Project administration: Lee HJ. Funding acquisition: Lee HJ. Approval of final manuscript: all authors.

\section{REFERENCES}

1. Kreiner DS, Shaffer WO, Baisden JL, Gilbert TJ, Summers JT, Toton JF, et al. An evidence-based clinical guideline for the diagnosis and treatment of degenerative lumbar spinal stenosis (update). Spine J 2013;13:734-43.

2. Fraser JF, Huang RC, Girardi FP, Cammisa FP Jr. Pathogenesis, presentation, and treatment of lumbar spinal stenosis associated with coronal or sagittal spinal deformities. Neurosurg Focus 2003;14:e6.

3. Geisser ME, Haig AJ, Tong HC, Yamakawa KS, Quint DJ, Hoff JT, et al. Spinal canal size and clinical symptoms among persons diagnosed with lumbar spinal stenosis. Clin J Pain 2007;23:780-5.

4. Lee SY, Kim TH, Oh JK, Lee SJ, Park MS. Lumbar stenosis: a recent update by review of literature. Asian Spine J 2015;9:818-28.

5. Lurie JD, Tosteson TD, Tosteson A, Abdu WA, Zhao W, Morgan TS, et al. Long-term outcomes of lumbar spinal stenosis: eight-year results of the Spine Patient Outcomes Research Trial (SPORT). Spine (Phila Pa 1976) 2015;40:63-76.

6. De Schepper EI, Overdevest GM, Suri P, Peul WC, Oei EH, Koes BW, et al. Diagnosis of lumbar spinal stenosis: an updated systematic review of the accuracy of diagnostic tests. Spine (Phila Pa 1976) 2013;38:E46981.

7. Wassenaar M, van Rijn RM, van Tulder MW, Verhagen AP, van der Windt DA, Koes BW, et al. Magnetic resonance imaging for diagnosing lumbar spinal pathol- ogy in adult patients with low back pain or sciatica: a diagnostic systematic review. Eur Spine J 2012;21:2207.

8. Steurer J, Roner S, Gnannt R, Hodler J; LumbSten Research Collaboration. Quantitative radiologic criteria for the diagnosis of lumbar spinal stenosis: a systematic literature review. BMC Musculoskelet Disord 2011;12:175.

9. Mamisch N, Brumann M, Hodler J, Held U, Brunner F, Steurer J, et al. Radiologic criteria for the diagnosis of spinal stenosis: results of a Delphi survey. Radiology 2012;264:174-9.

10. American Association of Electrodiagnostic Medicine. Guidelines in electrodiagnostic medicine: somatosensory evoked potentials: clinical uses. Muscle Nerve Suppl 1999;8:S111-8.

11. Snowden ML, Haselkorn JK, Kraft GH, Bronstein AD, Bigos SJ, Slimp JC, et al. Dermatomal somatosensory evoked potentials in the diagnosis of lumbosacral spinal stenosis: comparison with imaging studies. Muscle Nerve 1992;15:1036-44.

12. Egli D, Hausmann O, Schmid M, Boos N, Dietz V, Curt A. Lumbar spinal stenosis: assessment of cauda equina involvement by electrophysiological recordings. J Neurol 2007;254:741-50.

13. Han TR, Kim JH, Paik NJ. Electrodiagnostic study in spinal stenosis. J Korean Acad Rehabil Med 1992;16:460-6.

14. Eltantawi GA, Hassan MM, Sultan HE, Elnekiedy AA, Naby HM. Somatosensory-evoked potentials as an add-on diagnostic procedure to imaging studies in patients with lumbosacral spinal canal stenosis. Alex J Med 2012;48:207-14.

15. Shen N, Wang G, Chen J, Wu X, Wang Y. Evaluation of degree of nerve root injury by dermatomal somatosensory evoked potential following lumbar spinal stenosis. Neural Regen Res 2008;3:1249-52.

16. Aminoff MJ, Goodin DS, Barbaro NM, Weinstein PR, Rosenblum ML. Dermatomal somatosensory evoked potentials in unilateral lumbosacral radiculopathy. Ann Neurol 1985;17:171-6.

17. Han TR. Considerations in measuring somatosensory evoked potential. J Korean Acad Rehabil Med 1993;17:151-6.

18. Liu X, Konno S, Miyamoto M, Gembun Y, Horiguchi G, Ito $\mathrm{H}$. Clinical usefulness of assessing lumbar somato- 
sensory evoked potentials in lumbar spinal stenosis: clinical article. J Neurosurg Spine 2009;11:71-8.

19. Sipola P, Leinonen V, Niemelainen R, Aalto T, Vanninen $\mathrm{R}$, Manninen $\mathrm{H}$, et al. Visual and quantitative assessment of lateral lumbar spinal canal stenosis with magnetic resonance imaging. Acta Radiol 2011;52:1024-31.

20. Strojnik T. Measurement of the lateral recess angle as a possible alternative for evaluation of the lateral recess stenosis on a CT scan. Wien Klin Wochenschr 2001;113 Suppl 3:53-8.

21. Essa ZM, Al-Hashimi AF, Nema IS. Dermatomal versus mixed somatosensory evoked potentials in the diagnosis of lumbosacral spinal canal stenosis. J Clin Neurophysiol 2018;35:388-98.

22. Kuittinen P, Sipola P, Aalto TJ, Maatta S, Parviainen
A, Saari T, et al. Correlation of lateral stenosis in MRI with symptoms, walking capacity and EMG findings in patients with surgically confirmed lateral lumbar spinal canal stenosis. BMC Musculoskelet Disord 2014;15:247.

23. Andreisek G, Deyo RA, Jarvik JG, Porchet F, Winklhofer SF, Steurer J, et al. Consensus conference on core radiological parameters to describe lumbar stenosis: an initiative for structured reporting. Eur Radiol 2014;24:3224-32.

24. Andreisek G, Imhof M, Wertli M, Winklhofer S, Pfirrmann CW, Hodler J, et al. A systematic review of semiquantitative and qualitative radiologic criteria for the diagnosis of lumbar spinal stenosis. AJR Am J Roentgenol 2013;201:W735-46. 